\title{
Influence of Body Temperature on Bispectral Index-Guided Anesthetic Management in Off-Pump Coronary Artery Bypass Grafting
}

\author{
Muralidhar Kanchi ${ }^{1}$ Priya Nair ${ }^{1} \quad$ Rudresh Manjunath \\ ${ }^{1}$ Department of Anaesthesiology \& Critical Care Medicine, Narayana \\ Institute of Cardiac Sciences, Narayana Health City, Bangalore, \\ Karnataka, India \\ ${ }^{2}$ Department of Anesthesia, University of Minnesota, Minneapolis, \\ Minnesota, United States
}

J Card Crit Care:2020;4:79-85

\author{
Kumar Belani²
}

Address for correspondence Muralidhar Kanchi, MBBS, MD (anaes), MBA, Narayana Institute of Cardiac Sciences, Narayana Hrudayalaya Hospitals \#258/A, Bommasandra Industrial Area Anekal taluk, Bangalore, Karnataka, 560 099, India (e-mail: muralidhar.kanchi.dr@narayanahealth.org,/ kanchirulestheworld@gmail.com).

\section{Abstract}

Keywords

- bispectral index

- cardiac anesthesia

- end-tidal concentration

- body temperature

- coronary artery bypass grafting
Background Perioperative hypothermia is not uncommon in surgical patients due to anesthetic-induced inhibition of thermoregulatory mechanisms and exposure of patients to cold environment in the operating rooms. Core temperature reduction up to $35^{\circ} \mathrm{C}$ is often seen in off-pump coronary artery bypass graft (OP-CABG) surgery. Anesthetic depth can be monitored by using bispectral (BIS) index. The present study was performed to evaluate the influence of mild hypothermia on the anesthetic depth using BIS monitoring and correlation of BIS with end-tidal anesthetic concentration at varying temperatures during OP-CABG.

Materials and Methods In a prospective observational study design in a tertiary care teaching hospital, patients who underwent elective OP-CABG under endotracheal general anesthesia, were included in the study. Standard technique of anesthesia was followed. BIS, nasopharyngeal temperature, and end-tidal anesthetic concentration of inhaled isoflurane was recorded every 10 minutes. The BIS was adjusted to between 45 and 50 during surgery.

Results There were 40 patients who underwent OP-CABG during the study period. The mean age was $51.2 \pm 8.7$ years, mean body mass index $29.8 \pm 2.2$, and mean left ventricular ejection fraction was $55.4 \pm 4.2 \%$. Anesthetic requirement as guided by BIS between 45 and 50 correlated linearly with core body temperature $(r=0.999$; $p<0.001)$. The mean decrease in the body temperature at the end of 300 minutes was $2.2^{\circ} \mathrm{C}$ with a mean decrease in end-tidal anesthetic concentration of $0.29 \%$. The reduction in end-tidal anesthetic concentration per degree decrease in temperature was $0.13 \%$. None of the patients reported intraoperative recall.

Conclusion In this study, BIS monitoring was used to guide the delivery concentration of inhaled anesthetic using a targeted range of 45 to 50. BIS monitoring allowed the appropriate reduction of anesthetic dosing requirements in patients undergoing OP-CABG without risk of awareness. There was a significant reduction in anesthetic requirements associated with reduction of core temperature. The routine use of BIS is recommended in OP-CABG to titrate anesthetic requirement during occurrence of hypothermia and facilitate fast-track anesthesia in this patient population. (c) 2020. Official Publication of The Simulation Society (TSS), accredited by International Society of Cardiovascular Ultrasound (ISCU).

This is an open access article published by Thieme under the terms of the Creative Commons Attribution-NonDerivative-NonCommercial-License, permitting copying and reproduction so long as the original work is given appropriate credit. Contents may not be used for commercial purposes, or adapted, remixed, transformed or built upon. (https://creativecommons.org/licenses/by-nc-nd/4.0/)

Thieme Medical and Scientific Publishers Pvt. Ltd., A-12, 2nd Floor, Sector 2, Noida-201301 UP, India 


\section{Introduction}

Perioperative hypothermia is a consequence of loss of thermoregulation and patient exposure to cold environment in the operating room (OR). As the surgery proceeds, especially if the duration of surgery is greater than 1 hour, there is a loss of body thermal energy to the environment and redistribution of heat resulting in hypothermia. In patients who undergo off-pump coronary artery bypass grafting (OP-CABG), additional factors that enhance heat loss from patients include open thoracic cavity and exposure of lower extremities for harvesting the saphenous vein. Depth of anesthesia is a dynamic balance between the effect site concentration of hypnotic and analgesic drugs and the intensity of surgical stimulation. The incidence of awareness in cardiac surgery as reported in recent literature varies from 1.14 to $1.5 \%$ in patients after general anesthesia and cardiopulmonary bypass (CPB) with a low-dose opioid balanced anesthesia technique. ${ }^{1,2}$ Monitors to evaluate sedation effects are now available to titrate the commonly used intravenous and inhalational anesthetics. One such monitor namely, bispectral (BIS) index, analyzes the phase and frequency interrelationships of the electroencephalogram and yields a number from 0 to 100 that appears to provide an objective measure of a patient's level of hypnosis and sedation. ${ }^{3}$ Previous studies have indicated that BIS-monitored anesthesia reduced the risk of awareness in at-risk adult surgical patients. ${ }^{4}$ Fast tracking has been successful in decreasing hospital costs without loss of patient safety ${ }^{5}$ and fast track cardiac anesthesia is commonly practiced with one of the aims being extubation within 8 hours postoperatively. ${ }^{6}$ The safety of this early extubation practice has been extensively documented during the past 20 years. $^{7}$ To achieve this targeting a BIS range of between 40 and 60 has been advocated for awareness prevention and avoidance of excessive anesthesia. ${ }^{8}$ Although hypothermia can decrease anesthetic requirements, most work was performed in animal studies and a few studies have quantified the magnitude of its effects in humans. ${ }^{9}$ Several studies evaluating different systems to prevent intraoperative hypothermia have been evaluated..$^{10,11}$ Despite the use of these systems, it is not uncommon for core temperature to decrease anywhere between 1.1 and $1.6^{\circ} \mathrm{C}$. We studied the influence of decrease in temperature on the requirement of anesthetic during OP-CABG using BIS monitoring titrated anesthetic delivery.

\section{Methods}

After clearance from the institutional review board and obtaining informed consent, patients undergoing OP-CABG were enrolled for the study in a prospective observational study design. Patients requiring extracorporeal circulation either electively or during the course of operation, patients with a poor left ventricular (LV) function (ejection fraction $[\mathrm{EF}]<40 \%$ ), patients with LV aneurysms, renal/ hepatic dysfunction, patients requiring preoperative/intraoperative intra-aortic balloon pump, presence of unstable angina, carotid stenosis, history of cerebrovascular accident, excessive alcohol intake, and drug abuse were excluded from the study. Standard anesthetic and surgical techniques were used (details in the -Appendix 1). In brief, antihypertensive and antianginal medications were continued until the morning of surgery. Preanesthesia medication consisting of oral 10 mg of diazepam on the night prior to surgery and approximately 2 hours prior to anesthesia and surgery was administered. After arrival to the OR monitoring of electrocardiogram (5 lead) with automated ST segment analysis (Marquette solar 5000, GE Medical system, Milwaukee, Wisconsin, United States), noninvasive blood pressure, pulse oximetry, and a BIS sensor (BIS quatro 186-0106, model A-2000, Aspect Medical System, Natick, Massachusetts, United States) was applied. A $16-G$ intravenous cannula was inserted in the dorsum of the right hand and an 18-G, 9-cm intraarterial cannula was introduced into the right femoral artery for monitoring of the arterial pressure and obtaining arterial blood for analysis. General anesthesia was induced while the patient breathed $100 \% \mathrm{O}_{2}$ by facemask, using a combination of fentanyl $2 \mathrm{mcg} / \mathrm{kg}$, midazolam $100 \mathrm{mcg} / \mathrm{kg}$, and a sleep dose of propofol $(1-2 \mathrm{mg} / \mathrm{kg})$. Endotracheal intubation was performed after administration of pancuronium bromide at a dose of $0.15 \mathrm{mg} / \mathrm{kg}$ and mechanical ventilation to achieve normocarbia with end-tidal carbon dioxide monitoring was initiated. General anesthesia was maintained with isoflurane using a low-flow technique (fresh gas flow of $1 \mathrm{~L} / \mathrm{min}$ ) using a circle system delivered by an anesthesia machine (Excel 210 SE, Datex Ohmeda, Madison, Michigan, United States); the breathing circuit was connected to a humid-vent at the patient end. Delivered isoflurane (fraction of inhaled and exhaled concentration) was measured using the smart anesthetic gas monitoring system (CARESCAPE Monitor 650, GE Healthcare Finland Oy, Helsinki, Finland). Patient was provided with warm airflow at 40 degrees (Bair Hugger Warming Unit, Model 505, 3M, St. Paul, Minnesota, United States) and warm intravenous fluids. Temperature was recorded using a nasopharyngeal probe. The BIS was maintained between 45 and 50 during the entire procedure. BIS, temperature, heart rate, arterial oxygen saturation, and endtidal anesthetic (ETAC) and exhaled carbon dioxide concentration were recorded every 30 minutes. The BIS score was not recorded during the brief confounding signals such as during the use of electrocautery. After completion of the procedure, the patients were extubated as soon as the criteria were met in the surgical intensive care unit (ICU). All patients were interviewed 18 hours following extubation when they were alert and cooperative using a modified Brice questionnaire $^{12}(-$ Table 1$)$.

\section{Statistical Methods}

Data was analyzed by using SPSS statistical software for windows (ver.15, SPSS Inc, Chicago, Illinois, United States). Continuous data are presented in terms of mean and standard deviation, while categorical data are presented as number or percentage. Shapiro-Wilk test was used to test normality of the data distribution. Spearman's correlation test was used to test correlation between the parameters (BIS, ETAC, and temperature) at individual time points, whereas Pearson's 
correlation test was used to test the correlation between parameters over the time. A $p$-value less than 0.05 was considered statistically significant.

\section{Results}

A total of 40 patients underwent OP-CABG during the study period and all the 40 were included in the analysis. Of the 40 patients, $33(82.5 \%)$ were males. The mean age was $51.2 \pm 8.7$ (range: $30-70$ ) years. The nasopharyngeal temperature decreased during the first hour and then tapered with a maximum reduction occurring between the period 31 minutes to 60 minutes. A similar reduction in ETAC was also noted during the same time period with a maximum reduction occurring between 31 and 60 minutes. There was a 2 correlation between decrease in core temperature and ETAC $(r=0.999 ; p<0.001)$ over the time ( - Table 2). The mean BIS index at 0 minutes (prior to induction) was $93.7 \pm 3$ ( - Table 3 ). The BIS mean ranged from

Table 1 Modified Brice questionnaire used in the study Each patient was asked a standard set of questions (similar to Brice questionnaire) $)^{13}$

1. What was the last thing you remember before surgery?

2. What was the next thing you remember?

3. Can you remember anything in between the two periods?

4. Did you have any dreams during the operation?

If the patient indicated that he or she did not have any explicit memory of intraoperative events, no further questions were asked if the patient had any explicit memory the following subquestions will be asked:

5. What did you notice; sounds, touch, pain, paralysis?

6. How long did it last?

7. Have there been any consequences for you?

If patient suggested that he/she suffered from awareness under anesthesia, he/she was visited by the senior author and the attending anesthesiologist (consultant anesthesiologist) to discuss and explain the perioperative events and answer patient's questions sympathetically and refer the patient for psychological counseling, if necessary.
43.4 to 50.8 to 30 to 300 minutes at which time the surgery was completed. The maximum reduction in core temperature was $2.2^{\circ} \mathrm{C}$ and this was associated with a reduction in ETAC of $0.29 \%$. The change in ETAC per degree decrease in core temperature was a decrement of $0.131 \%$.

\section{Discussion}

Perioperative hypothermia is not an uncommon occurrence during OP-CABG. Median sternotomy and low ambient temperature in the OR promotes heat loss under anesthesia. Almost the entire body is exposed during CABG as the leg veins are harvested from the lower extremity. Anesthesia-induced alteration in thermoregulation, wide surgical exposure, cold ambient OR temperature, transfusion of intravenous fluids, and relatively long duration of surgery are factors responsible for heat loss during OP-CABG. A decrease in temperature occurs despite warming blanket underneath the patient's body, warm intravenous fluids, and warming the inspired gases. This study demonstrated a decrease of the body temperature of up to $2.2^{\circ} \mathrm{C}$ during OP-CABG. The occurrence of intraoperative mild hypothermia intraoperatively was associated with a reduction in anesthesia requirement as guided by use of the BIS index. In a study addressing the impact of postoperative hypothermia on outcomes in CABG, Yu et al observed that hypothermia was associated with low body mass index, elderly age group, female sex, low preoperative hematocrit value, high LVEF, and short CPB time. ${ }^{13}$ There were no differences in the postoperative outcomes in those who had hypothermia and those who did not. There is published data on perioperative hypothermia and negative outcomes in patients undergoing noncardiac surgery, but there is a paucity of literature and inconsistent data that look at effects of postoperative hypothermia in patients undergoing cardiac surgery. Hannan et al performed a retrospective study in patients undergoing OP-CABG and similarly found that patients who left the OR mildly hypothermic had significantly higher rates of respiratory failure and unplanned

Table 2 Correlation between end-tidal concentration and temperature across the procedure at different time intervals

\begin{tabular}{|l|l|l|l|l|}
\hline & $\begin{array}{l}\text { End-tidal anesthetic concentration }(\%) \text { (mean } \pm \\
\text { SD) }\end{array}$ & $\begin{array}{l}\text { Temperature } \\
\left({ }^{\circ} \mathrm{C}\right) \\
(\text { mean } \pm \text { SD) }\end{array}$ & $\begin{array}{l}\text { Correlation } \\
\text { coefficient } \\
(r)\end{array}$ & \begin{tabular}{l}
$p-$ Value \\
\hline $30 \mathrm{~min}$
\end{tabular} \\
\hline $60 \mathrm{~min}$ & $0.978 \pm 0.04$ & $36.79 \pm 0.43$ & 0.228 & 0.158 \\
\hline $90 \mathrm{~min}$ & $0.934 \pm 0.03$ & $36.35 \pm 0.52$ & 0.277 & 0.084 \\
\hline $120 \mathrm{~min}$ & $0.898 \pm 0.03$ & $35.97 \pm 0.44$ & 0.203 & 0.209 \\
\hline $150 \mathrm{~min}$ & $0.867 \pm 0.03$ & $35.62 \pm 0.49$ & 0.139 & 0.391 \\
\hline $180 \mathrm{~min}$ & $0.841 \pm 0.03$ & $35.37 \pm 0.48$ & 0.198 & 0.222 \\
\hline $210 \mathrm{~min}$ & $0.813 \pm 0.05$ & $35.15 \pm 0.44$ & 0.111 & 0.496 \\
\hline $240 \mathrm{~min}$ & $0.791 \pm 0.04$ & $34.94 \pm 0.46$ & 0.210 & 0.186 \\
\hline $270 \mathrm{~min}$ & $0.776 \pm 0.04$ & $34.75 \pm 0.42$ & 0.319 & $0.045^{\mathrm{a}}$ \\
\hline $300 \mathrm{~min}$ & $0.761 \pm 0.05$ & $34.69 \pm 0.35$ & 0.313 & $0.049^{\mathrm{a}}$ \\
\hline
\end{tabular}


Table 3 Mean core body temperature, end-tidal anesthetic concentration, and bispectral index at various time intervals

\begin{tabular}{|l|l|l|l|}
\hline Time & $\begin{array}{l}\text { Temperature }\left(\mathbf{C}^{\circ}\right) \\
(\text { mean } \pm \text { SD) }\end{array}$ & $\begin{array}{l}\text { End-tidal anesthetic concentration (\%) } \\
(\text { mean } \pm \text { SD) }\end{array}$ & $\begin{array}{l}\text { Bispectral index } \\
(\text { mean } \pm \text { SD) }\end{array}$ \\
\hline 0 min & - & - & $93.7 \pm 3.0$ \\
\hline $30 \mathrm{~min}$ & $36.79 \pm 0.43$ & $1.045 \pm 0.09$ & $43.42 \pm 2.05$ \\
\hline $60 \mathrm{~min}$ & $36.35 \pm 0.52$ & $0.978 \pm 0.04$ & $44.72 \pm 2.27$ \\
\hline $90 \mathrm{~min}$ & $35.97 \pm 0.44$ & $0.934 \pm 0.03$ & $46.50 \pm 3.23$ \\
\hline $120 \mathrm{~min}$ & $35.62 \pm 0.49$ & $0.898 \pm 0.03$ & $47.25 \pm 3.44$ \\
\hline $150 \mathrm{~min}$ & $35.37 \pm 0.48$ & $0.867 \pm 0.03$ & $49.22 \pm 4.18$ \\
\hline $180 \mathrm{~min}$ & $35.15 \pm 0.44$ & $0.841 \pm 0.03$ & $50.55 \pm 5.22$ \\
\hline $210 \mathrm{~min}$ & $34.94 \pm 0.46$ & $0.813 \pm 0.05$ & $50.30 \pm 4.54$ \\
\hline $240 \mathrm{~min}$ & $34.75 \pm 0.42$ & $0.791 \pm 0.04$ & $50.20 \pm 5.33$ \\
\hline $270 \mathrm{~min}$ & $34.69 \pm 0.35$ & $0.776 \pm 0.04$ & $45.57 \pm 4.94$ \\
\hline $300 \mathrm{~min}$ & $34.59 \pm 0.35$ & $0.761 \pm 0.05$ & $50.77 \pm 3.99$ \\
\hline
\end{tabular}

Abbreviations: ETAC, end-tidal anesthetic concentration; SD, standard deviation.

Note: Total change in temperature from baseline till 300 minutes was $2.2 \pm 0.52^{\circ} \mathrm{C}$. Total change in ETAC from baseline till 300 minutes was $0.29 \pm 0.12 \%$.

re-exploration for bleeding compared with normothermic patients. ${ }^{14}$ In contrast, in a retrospective study by Karalapillai et al that determined patient outcomes after all elective cardiac surgery, not just CABG, the authors demonstrated that transient postoperative hypothermia lasting $<24$ hours was not independently associated with increased hospital mortality ${ }^{15}$ Likewise, a study by Nathan et al where patients undergoing on-pump CABG were randomly assigned to having their core temperature maintained at either $37^{\circ} \mathrm{C}$ or $34^{\circ} \mathrm{C}$ throughout the intraoperative period, did not find significant differences in blood product utilization, intubation time, time in the hospital, myocardial infarction, or mortality between the two groups. ${ }^{16}$

Volatile anesthetic agents form an integral part of anesthesia for cardiac surgery. Intraoperative awareness remains one of the major concerns during cardiac surgery. By virtue of their amnestic properties, volatile anesthetic agents markedly decrease the incidence of awareness while allowing easy titratability of anesthetic depth. ${ }^{17,18}$ Depth of anesthesia is the interaction of two drug effects during clinical anesthesia, one drug effect involves hypnosis and the second effect involves analgesia. The hypnotic component can be induced by intravenous and inhaled anesthetics whereas analgesia component can be induced by opioids and local anesthetics. Several new monitors have been studied to assess the depth of anesthesia; among them, the BIS monitor (Bi-spectral Index, Aspect Medical System) has undergone extensive investigation. BIS utilizes processed electroencephalography (EEG) with advanced algorithms to display an index from 0 to $100 ; 100$ represents an awake state and 0 represents complete EEG inactivity. BIS index is presently one of the most extensively validated measures of depth of anesthesia. Titration of anesthetic agents by BIS allows precision in sedation without overdosing while minimizing awareness currently estimated at $0.2 \%$ in healthy patients undergoing general anesthesia and $1.14 \%$ in patients undergoing cardiac surgery. In the present study, the anesthesia

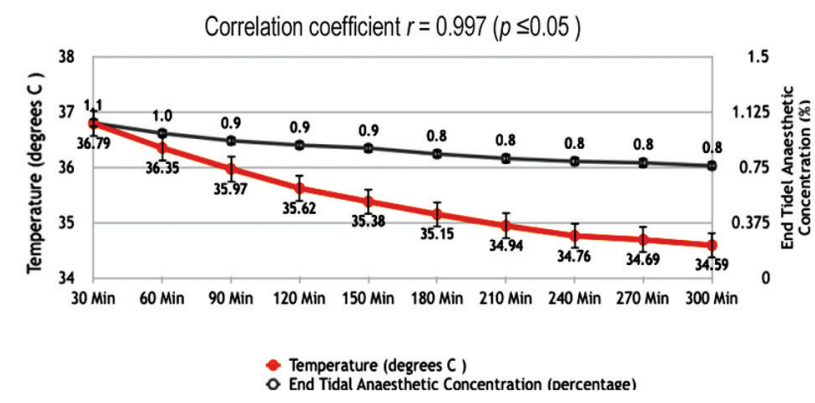

Fig. 1 Correlation between core body temperature and exhaled anesthetic concentration.

dose of isoflurane was guided by the BIS index titrated to a value between 40 and 60 to maintain a constant, moderate depth of anesthesia and allow rapid tracking and early recovery. During the intraoperative mild hypothermia, it is expected that there would be a reduction in anesthesia requirement as suggested by use of the BIS index. Utilizing the BIS values between 45 and 50 also ensured lack of recall in this group of patients. In a previous study done in adults undergoing on-pump CABG surgery, Liu et a ${ }^{9}$ found increasing and statistically significant isoflurane requirements as core temperature increased from $30^{\circ} \mathrm{C}$ to $37^{\circ} \mathrm{C}$ during rewarming from $\mathrm{CPB}$. In OP-CABG, we have demonstrated a positive correlation between temperature and ETAC ( $r=$ $0.999 ; p<0.01$ ). Hypothermia is known to decrease anesthetic requirements, our study corroborated this $\left(r^{2}=0.997\right.$;

-Fig. 1). In our study we found that to maintain the constant BIS value, isoflurane requirements decreased by $0.131 \%$ per degree Celsius decrease in core temperature. It is possible that the temperature-related increased solubility of inhaled anesthetics in the lipid layer of cells may explain the reduced need at the cellular level. By utilizing processed EEG monitoring one can decrease the avoidable overdose of anesthetic agents thus decreasing the likelihood of myocardial depression, arrhythmias, vasodilation, and need for 
inotropes or vasopressors. This will be particularly important in those with poor LV reserve as may be present in the elderly age groups.

In a study by Muralidhar et $\mathrm{al}^{19}{ }^{19}$ the effect of BIS-guided anesthesia on the requirement of anesthetic agents in OP-CABG was studied. It was observed that there was a statistically significant reduction in the anesthetic requirement in the BIS-guided groups. Time to tracheal extubation was significantly reduced in the group. No patient had explicit memory of any event from the induction of anesthesia to recovery of consciousness in the cardiac surgical ICU. In the present study too, BIS was used to maintain a constant, moderate depth of anesthesia and the anesthesia dose was adjusted to obtain a BIS value of 40 to 60 . There was a significant reduction in isoflurane requirement and none of the 40 patients had any recall of intraoperative events similar to the previous study. Song et al have shown that titrating desflurane and sevoflurane using a BIS monitor decreased volatile agent dose and contributed to a faster emergence from anesthesia in outpatients undergoing laparoscopic tubal ligation procedures. ${ }^{20}$ Yet another group reported significantly improved recovery with the use of BIS-titrated inhaled agent during surgical care. ${ }^{21}$ The reduced anesthetic drug requirements during OP-CABG can be extrapolated to less myocardial depression, less decrease in system vascular resistance, reduced arrhythmias, decreased need for inotropic support, or vasoconstrictor medication. Precision of anesthetic drug usage in cardiac surgery is of great importance in patients with poor LV function, in elderly population, and in high-risk groups. Perianesthetic unintentional hypothermia produces multiple effects with few beneficial and few other potentially severe side effects. The benefits of hypothermia are protection against cerebral ischemia but adverse effects of hypothermia are potentially detrimental that may be associated with increased morbidity and mortality in patients undergoing long-duration noncardiac surgery. The effects of unintentional hypothermia include increased risk of surgical site infection, impaired coagulation, perioperative bleeding, transfusion requirements, and myocardial ischemia in noncardiac surgery. The effects of hypothermia of $<36.0^{\circ} \mathrm{C}$ in patients who underwent CABG were no different from the normothermic group. The lack of demonstrable difference in outcomes between the normothermic and mildly hypothermic groups may be related to the fact that the opposing effects are cancelled out.

\section{Limitation of the Study}

The limitation of our study was a small sample size (40 patients) and is a single-center study.

\section{Conclusion}

Perioperative hypothermia is not an infrequent occurrence during OP-CABG surgery. The use of BIS monitoring allows the safe reduction of inhaled agents to preset goals, prevents anesthesia overdose and associated side effects without increasing intraoperative awareness, and allows fast tracking of patients undergoing OP-CABG surgery.

\section{Conflict of Interest}

None declared.

\section{References}

1 Phillips AA, McLean RF, Devitt JH, Harrington EM. Recall of intraoperative events after general anaesthesia and cardiopulmonary bypass. Can J Anaesth 1993;40(10):922-926

2 Ranta S, Jussila J, Hynynen M. Recall of awareness during cardiac anaesthesia: influence of feedback information to the anaesthesiologist. Acta Anaesthesiol Scand 1996;40(5):554-560

3 Sigl JC, Chamoun NG. An introduction to bispectral analysis for the electroencephalogram. J Clin Monit 1994;10(6):392-404

4 Myles PS, Leslie K, McNeil J, Forbes A, Chan MT. Bispectral index monitoring to prevent awareness during anaesthesia: the B-Aware randomised controlled trial. Lancet 2004;363(9423):1757-1763

5 Cohn LH, Rosborough D, Fernandez J. Reducing costs and length of stay and improving efficiency and quality of care in cardiac surgery. Ann Thorac Surg 1997;64(6, Suppl):S58-S60, discussion S80-S82

6 Cheng DC, Karski J, Peniston C, et al. Early tracheal extubation after coronary artery bypass graft surgery reduces costs and improves resource use. A prospective, randomized, controlled trial. Anesthesiology 1996;85(6):1300-1310

7 Larson SL, Schimmel CH, Shott S, Myers PB, Foy BK. Influence of fast-track anesthetic technique on cardiovascular infusions and weight gain. J Cardiothorac Vasc Anesth 1999;13(4):424-430

8 Monk TG, Saini V, Weldon BC, Sigl JC. Anesthetic management and one-year mortality after noncardiac surgery. Anesth Analg 2005;100(1):4-10

9 Liu M, Hu X, Liu J. The effect of hypothermia on isoflurane MAC in children. Anesthesiology 2001;94(3):429-432

10 Hofer CK, Worn M, Tavakoli R, et al. Influence of body core temperature on blood loss and transfusion requirements during off-pump coronary artery bypass grafting: a comparison of 3 warming systems. J Thorac Cardiovasc Surg 2005;129(4):838-843

11 Matsukawa T, Sessler DI, Sessler AM, et al. Heat flow and distribution during induction of general anesthesia. Anesthesiology 1995;82(3):662-673

12 Brice DD, Hetherington RR, Utting JE. A simple study of awareness and dreaming during anaesthesia. $\mathrm{Br}$ Anaesth 1970;42(6):535-542

13 Yu P-J, Cassiere HA, Kohn N, Mattia A, Hartman AR. Impact of postoperative hypothermia on outcomes in coronary artery bypass surgery patients. J Cardiothorac Vasc Anesth 2017;31(4):1257-1261

14 Hannan EL, Samadashvili Z, Wechsler A, et al. The relationship between perioperative temperature and adverse outcomes after off-pump coronary artery bypass graft surgery. J Thorac Cardiovasc Surg 2010;139(6):1568-1575.e1

15 Karalapillai D, Story D, Hart GK, et al. Postoperative hypothermia and patient outcomes after elective cardiac surgery. Anaesthesia 2011;66(9):780-784

16 Nathan HJ, Parlea L, Dupuis JY, et al. Safety of deliberate intraoperative and postoperative hypothermia for patients undergoing coronary artery surgery: a randomized trial. J Thorac Cardiovasc Surg 2004;127(5):1270-1275

17 Joshi GP. Inhalational techniques in ambulatory anesthesia. Anesthesiol Clin North America 2003;21(2):263-272

18 Dowd NP, Cheng DC, Karski JM, Wong DT, Munro JA, Sandler AN. Intraoperative awareness in fast-track cardiac anesthesia. Anesthesiology 1998;89(5):1068-1073, discussion 9A

19 Muralidhar K, Banakal S, Murthy K, Garg R, Rani GR, Dinesh R. Bispectral index-guided anaesthesia for off-pump 
coronary artery bypass grafting. Ann Card Anaesth 2008;11(2): 105-110

20 Song D, Joshi GP, White PF. Titration of volatile anesthetics using bispectral index facilitates recovery after ambulatory anesthesia. Anesthesiology 1997;87(4):842-848
21 Gan TJ, Glass PS, Windsor A, et al; BIS Utility Study Group. Bispectral index monitoring allows faster emergence and improved recovery from propofol, alfentanil, and nitrous oxide anesthesia. Anesthesiology 1997;87(4):808-815 